\title{
Applying Deliberate Practice to Facilitate Schema Acquisition in Learning Introductory Mechanics
}

\section{Dr. Yan Tang, Embry-Riddle Aeronautical University - Daytona Beach}

Dr. Yan Tang is an associate professor of mechanical engineering at Embry-Riddle Aeronautical University in Daytona Beach, Fla. Her current research in engineering education focuses on cognitive load theory, deliberate practice, and effective pedagogical practices. Her background is in dynamics and controls.

\section{Dr. Haiyan Bai, University of Central Florida}

Haiyan Bai, PhD., is an Associate Professor of Quantitative Research Methodology in the College of Education and Human Performance at the University of Central Florida. Her interests include resampling method, propensity score analysis, research design, measurement and evaluation, and the applications of statistical methods in educational research and behavioral sciences. She is actively involved educational and social science research projects. Dr. Bai has published books and many professional articles in refereed national and international journals. She has won several competitive awards at the University of Central Florida for her excellent teaching and research. Dr. Bai also served on several professional journal editorial boards, such as Journal of Experimental Education, Frontiers in Quantitative Psychology and Measurement, and Journal of Data Analysis and Information Processing. She is also the Fellow of the Academy for Teaching, Learning, and Leadership and the Faculty Fellow at The University of Central Florida.

\section{Dr. Richard Catrambone, Georgia Institute of Technology}

Richard Catrambone is a Professor in the School of Psychology at the Georgia Institute of Technology. He received his B.A. from Grinnell College and his Ph.D. in Experimental Psychology from the University of Michigan.

The question Catrambone likes to ask-and the thread that runs through the projects he does alone and in collaboration with others-is: What does someone need to know in order to solve novel problems or carry out tasks within a particular domain?

Catrambone's research interests include problem solving, educational technology, and human-computer interaction. He is particularly interested in how people learn from examples in order to solve problems in domains such as algebra, probability, and physics. He explores how to create instructional materials that help learners understand how to approach problems in a meaningful way rather than simply memorizing a set of steps that cannot easily be transferred to novel problems. He researches the design of teaching and training materials-including software and multimedia environments-based on cognitive principles that help students learn basic tasks quickly and promote transfer to novel problems. He uses task analysis to identify what someone needs to know in order to solve problems or carry out tasks in a domain and then to use the results of the task analysis to guide the construction of teaching and training materials/environments.

Catrambone has served on the Cognitive Science Society governing board from 2011-2016 and was chair of the Society in 2015. He was co-chair of the Cognitive Science Conference in 2010. He has served as a consulting editor for the Journal of Educational Psychology (1/2008 - 12/2011), the Journal of Experimental Psychology: Learning, Memory, and Cognition (6/2000 - 12/2001 and 1/2009 - 12/2009), the Journal of Experimental Psychology: Applied (1/2001 - 12/2007), and the Journal of Experimental Psychology: General (6/2000 - 12/2001). He has published his research in journals such as the Journal of Experimental Psychology: General; Journal of Experimental Psychology: Learning, Memory, and Cognition; Journal of Experimental Psychology: Applied; Memory \& Cognition; Journal of Educational Psychology; Human-Computer Interaction; Human Factors; and other basic and applied journals. He has also served on grant review panels for a variety of funding agencies including the National Science Foundation and the Institute of Education Sciences (U.S. Department of Education). 


\title{
Applying Deliberate Practice to Facilitate Schema Acquisition in Learning Introductory Mechanics
}

\begin{abstract}
Learning is usually conceptualized as a process during which new information is processed in working memory to form knowledge structures called schemas, which are stored in long-term memory. Practice plays a critical role in developing schemas through learning by doing. Contemporary expertise development theories have highlighted the influence of deliberate practice (DP) on achieving exceptional performance in sports, music, and different professional fields. Concurrently, there is an emerging method for improving learning efficiency by combining deliberate practice with cognitive load theory (CLT), a cognition-architecture-based theory for instructional design.
\end{abstract}

Mechanics serves as a foundation for most branches of engineering. It develops problem-solving skills and consolidates understanding of other subjects, such as applied mathematics and physics. Mechanics is a challenging subject. Students need to understand the governing principles to gain conceptual knowledge and acquire procedural knowledge to apply these principles to solve problems. Due to the difficulty in developing conceptual and procedural knowledge, mechanics courses are among those which receive high DFW rates (percentage of students receiving a grade of $\mathrm{D}$ or $\mathrm{F}$ or withdrawing from a course) and students are more likely to leave engineering after taking mechanics courses. Since deliberate practice can help novices develop good representations of the knowledge needed to produce superior problem-solving performance, this study was designed to evaluate how deliberate practice helps students learn mechanics during the process of schema acquisition and consolidation. Considering cognitive capacity limitations, we applied cognitive load theory to develop deliberate practice to help students build declarative and procedural knowledge without exceeding their working memory limitations.

We evaluated the effects of three practice strategies based on CLT on the schema acquisition and consolidation in two mechanics courses (i.e., Statics and Dynamics). Examples and assessment results are provided to evaluate the effectiveness and challenges of the practice strategies.

\section{Introduction}

Learning is usually conceptualized as a process during which new information is processed in working memory to form knowledge structures called schemas, which are stored in long-term memory. Practice plays a critical role in developing schemas through learning-by-doing. Contemporary expertise development theories have highlighted the influence of deliberate practice (DP) on achieving exceptional performance in sports, music, and different professional fields. Concurrently, there is an emerging method for improving learning efficiency by combining deliberate practice with cognitive load theory (CLT), a cognition-architecture-based theory for instructional design.

Mechanics is a foundation for most branches of engineering. It serves to develop problem-solving skills and consolidate understanding of other subjects, such as applied mathematics and physics. Mechanics is a challenging subject. Students need to understand governing principles to gain conceptual knowledge and acquire procedural knowledge to apply these principles to solve 
problems. Due to the difficulty in developing conceptual and procedural knowledge, mechanics courses are among those which receive high DFW rates (percentage of students receiving a grade of D or F or withdrawing from a course) and students are more likely to leave engineering after taking mechanics courses. Since deliberate practice can help novices develop good representations of the knowledge needed to produce superior problem-solving performance, this study was designed to evaluate how deliberate practice helps students learn mechanics during the process of schema acquisition and consolidation. Considering cognitive capacity limitations, we applied cognitive load theory to develop deliberate practice to help students build declarative and procedural knowledge without exceeding their working memory limitations.

In this paper, we will first introduce the theoretic frameworks on which this study is based: CLT and DP, followed by a brief description of the Four Component Instructional Design (4C/ID), the instructional design model we used to develop deliberate practice materials. Then we will show how we followed the 4C/ID to develop practice problems. Examples and assessment results are provided to evaluate the effectiveness and challenges of the practice strategies.

\section{Theoretic Frameworks: Cognitive Load Theory and Deliberate Practice}

Based on human cognitive learning processes, CLT has provided a comprehensive set of instructional principles [1]. Since our working memory can process only $7 \pm 2$ individual items at one time, learning is hindered if the information to be processed exceeds those limits. CLT provides specific instructional guidelines which adjust learning materials based on limited working memory capacity.

Cognitive load imposed on working memory can be divided into two categories: intrinsic cognitive load and extraneous cognitive load [1]. As indicated by the names, intrinsic cognitive load refers to the mental work determined by the intrinsic nature of the information that the learner needs to acquire while extraneous cognitive load refers to the mental work which is unnecessary and extraneous to learning goals (such as an interface for a computer-based instructional environment). Since intrinsic load is related to the complexity of learning materials, difficult learning materials, if other factors (e.g., learners' prior knowledge) being held constant, will of course impose more cognitive load than easy ones. Generally, intrinsic load is a fixed quality of the material to be learned. By contrast, a teacher or designer should strive to reduce extraneous load so that limited mental resources can be committed to intrinsic load to maximize learning.

Deliberate practice (DP) emerged in several studies on the acquisition of expertise three decades ago. DP is a particular type of practice with the deliberate intention of developing a specific skill that is beyond the learner's current ability. As shown in Figure 1, DP includes mechanisms for monitoring and guiding continuous improvement of specific aspects of performance [2]. Ericsson et al. [3] found that exceptional performance was caused by practice strategy rather than innate ability. However, most research has focused on the influence of DP on advanced skill development among experts and much less is known about its influence on novices [4-5].

Studies have shown improved learning by applying CLT into developing DP strategies in medical education ([6-8]). CLT exploits the limits and strengths of the human learning processes to provide guidelines for more efficient learning. Over a 30-year period, CLT has generated a variety of 
instructional procedures through replicable, randomized, and controlled experiments. Instructional procedures developed by CLT efficiently generate and increase schemas in long-term memory. In this study, we apply CLT to design DP which facilitates novice learning to achieve effective and efficient learning.

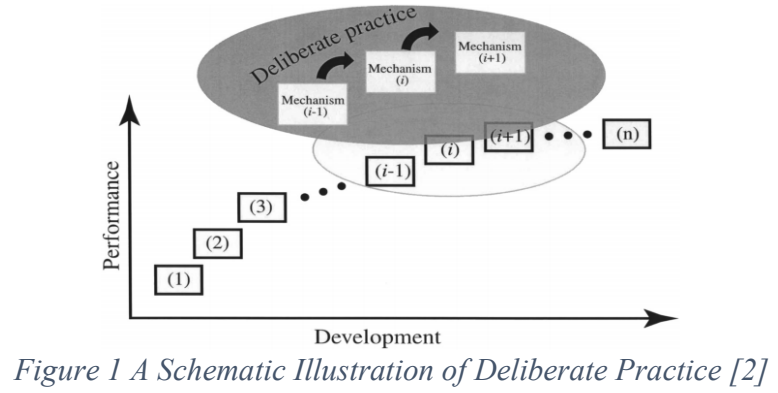

\section{Instructional Design Model: 4C/ID}

The Four Component/Instructional Design (4C/ID) is, to the best of our knowledge, the only systematic instructional design model that has integrated CLT and DP [9]. Based on a broad body research on teaching and training of complex skills, this instructional design model has evolved over 25 years. The four components refer to four basic interrelated learning elements: learning tasks, supportive information for non-routine learning tasks, procedural information for routine learning tasks, and part-task practice for achieving automaticity of constituent skills to complete learning tasks [9].

The 4C/ID consists of 10 design steps detailing the design guidelines for the four components. The first three steps aim at choosing appropriate learning tasks, developing performance assessment, and creating a proper sequence of learning tasks to maximize learning efficiency. Since learning tasks can be generally divided into non-routine and routine tasks, the next six steps are dedicated to design support and information to facilitate learning without overloading working memory. The last step is to help learners to achieve the required level of fluency to complete certain learning tasks.

Due to the intrinsic difficulty of dynamics, we adopted the 4C/ID in developing practice problems to facilitate learning in dynamics. In this paper, we will show how we applied the 4C/ID to design practice problems to help students learn one-dimensional kinematics to evaluate the effectiveness of this instructional design method.

\section{Applying 4C/ID in Teaching One-Dimensional Kinematics}

One-dimensional kinematics is the first topic in dynamics. This topic is first introduced in introductory physics before dynamics, though it mainly deals with motion with constant acceleration. Many students get used to solving this type of problems with the kinematics formula $v_{1}=v_{0}+a t$ without realizing that this equation works only for motion with constant acceleration. 
To address this knowledge deficiency and teach students the proper thought process, the $4 \mathrm{C} / \mathrm{ID}$ was used to develop practice problems.

Step 1: Design learning tasks. Learning tasks here refer to one-dimensional kinematics problems students need to learn how to solve. Based on the given kinematics process information, the following learning task classes are created as shown in Figure 2. Kinematics process refers to information which is used to describe the motion (e.g., $s(t)=3 t^{2} \mathrm{ft}$ or $a(v)=-4 v^{2} \mathrm{~m} / \mathrm{s}^{2}$ ). We mainly focus on motion with acceleration given as a function as this is a critical foundation for particle kinetics. Depending on the argument of the acceleration function, we created three learning task classes. For example, the first learning task class includes only problems with acceleration or angular acceleration given as a function of time. Problems with varying levels of difficulty and variations in contexts are selected.

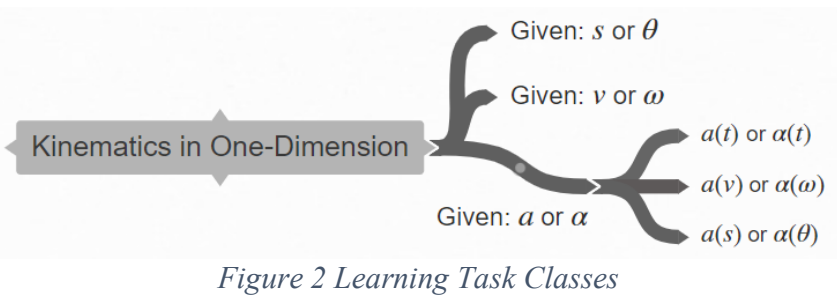

Step 2: Design performance assessment. To determine performance assessments, we have conducted skill decomposition by splitting skills required to solve these problems into constituent skills. For example, if acceleration is given to describe a motion, we need to apply a definite integral to solve the problem. First, students should be able to interpret problems or correctly represent givens and what they need to find. Students should also be able to apply separation of variables and/or the chain rule to integrate acceleration. Since we require students to set up a system of equations before finding the numerical solutions, students need to recognize unknowns to ensure setting up an equal number of equations for unknowns. Therefore, for these types of problems, four constituent skills are selected: 1 . represent givens and finds; 2 . apply separation of variables; 3 . apply the chain rule; and 4 . recognize unknowns.

Step 3: Sequence learning tasks. The learning tasks are organized sequentially from simple to complex levels to avoid overloading working memory while imposing desired difficulty when students' knowledge and skills increase. Consider the same example from Step 2. The simplest examples would be problems resulting in a solution with only one equation by applying separation of variables to $a(t)=\frac{d v}{d t}$. More complex problems would be a solution with a system of equations. The complexity level can also be determined by the number of required component skills students need to learn.

Step 4: Design supportive information. Although the learning tasks are presented in sequential complexity, the simplest problems may impose challenges to students when they are new to the learning material. We need to provide supportive information to guide students through the problem-solving process. For example, we could provide a systematic problem-solving process as shown in Figure 3 to serve as a scaffold to provide support. It is occasionally necessary to analyze students' common mistakes to develop guidelines to facilitate problem-solving. For instance, instead of applying separation of variables to integrate the acceleration, students often create the 
resulting equations based on their memory (e.g., $\int_{v_{0}}^{v_{1}} a(v) d v=\int_{t_{0}}^{t_{1}} d t$ rather than $\int_{v_{0}}^{v_{1}} \frac{1}{a(v)} d v=$ $\int_{t_{0}}^{t_{1}} d t$ ). For such situations, it would be beneficial to ask students to check the units on each side of the equation to determine whether the units match would be beneficial. Such supportive information is often overlooked by subject matter experts or the instructors, but it is essential for novice learners. Analyzing students' common mistakes will help develop the supportive information that facilitates learning.

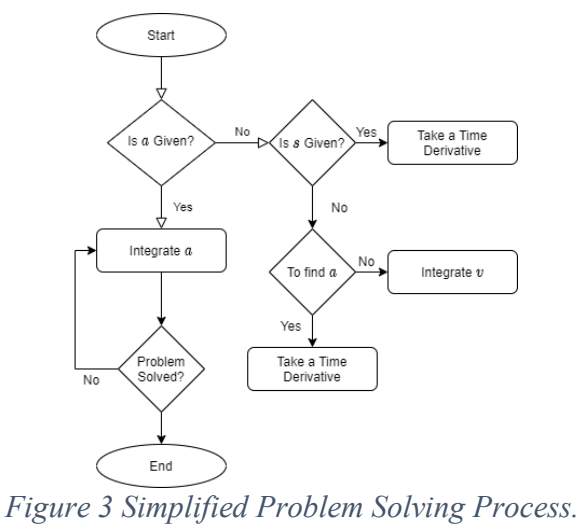

Step 5: Design part-task practice. Before students master all required prerequisite information or skills, solving a whole problem may impose a tremendous challenge. This situation often occurs in dynamics as many students do not have solid foundations to integrate all information together effectively. We develop part-task practice to help students acquire and consolidate required skills. For example, students often have trouble applying separation of variables. Practice problems focusing on this single step helps them understand how separation of variables is applied for different scenarios (e.g., $a(t)$ or $a(v))$ and how to identify the mistakes by checking units). This strategy not only helps reduce the mental load on students' working memory, it could also improve learning efficiency. Given 10 minutes of practice time, students may not be able to solve a whole problem, but they can understand how separation of variables is applied by studying a couple of

examples such as $a(t) d t=d v$ or $d t=\frac{1}{a(v)} d v$ followed by practicing on similar problems with just the step on separation of variables. Depending on students' prior knowledge, part-task practice problems and a practice schedule can be developed to help students achieve automaticity of each constituent skill before they integrate all skills together to solve the whole problem.

\section{Results and Discussions}

A quasi-experimental research design was used to study the program effectiveness with two sections of ES204 Dynamics at Embry-Riddle Aeronautical University in Spring 2021. The two sections were randomly assigned to the treatment or control group (random cluster assignment of intact group). In the total sample of 80 , there were 39 students in the treatment group and 41 students in the control group. Data were collected after IRB approval in both universities of the researchers. Analysis of covariance (ANCOVA) test results revealed that the mean of post-test scores (measured by the total scores of 12 objective test items) of the treatment group $(M=10.44$, $S D=1.68)$ were significantly higher than the control group $(M=9.50, S D=2.75)$ (See Table 1$)$ 
with $F(1,77)=5.79, p=.019<.05$ (See Table 2) while controlling for the effect of student GPAs which was significant $(F(1,77)=16.08, p=.001)$ (See Table 2$)$.

Table 1. Descriptive Statistics

\begin{tabular}{lr|c|c}
\hline Groups & Mean & SD & $N$ \\
\hline Control & 9.4959 & 2.74583 & 41 \\
\hline Treat & 10.4444 & 1.68296 & 39 \\
\hline Total & 9.9583 & 2.32541 & 80 \\
\hline
\end{tabular}

Table 2. Tests of Between-Subjects Effects

Dependent Variable: Total posttest scores of homework

\begin{tabular}{lr|r|r|r|r|r}
\hline \multicolumn{1}{|c}{ Source } & $\begin{array}{c}\text { Type III } \\
\text { Sum of } \\
\text { Squares }\end{array}$ & $d f$ & $\begin{array}{c}\text { Mean } \\
\text { Square }\end{array}$ & $F$ & $P$ & $\eta^{2}$ \\
\hline $\begin{array}{l}\text { Corrected } \\
\text { Model }\end{array}$ & $88.683^{\mathrm{a}}$ & 2 & 44.342 & 10.086 & .000 & .208 \\
\hline Intercept & 8.609 & 1 & 8.609 & 1.958 & .166 & .025 \\
\hline GPA & 70.701 & 1 & 70.701 & 16.082 & .000 & .173 \\
\hline Groups & 25.434 & 1 & 25.434 & 5.785 & .019 & .070 \\
\hline Error & 338.511 & 77 & 4.396 & & & \\
\hline Total & 8360.667 & 80 & & & & \\
\hline $\begin{array}{l}\text { Corrected } \\
\text { Total }\end{array}$ & 427.194 & 79 & & & & \\
\hline
\end{tabular}

a. R Squared $=.208$ (Adjusted R Squared $=.187)$

The results provided preliminary evidence that deliberate practice developed with 4C/ID facilitates schema acquisition in learning introductory mechanics. Note that, in this analysis, we controlled the effect of students' GPAs as there was significant difference between the two groups. There might be other influencing factors (e.g., demographic data) that could confound the results. In the future study, we will collect more data to evaluate where there are any subgroup differences or covariance to be controlled.

\section{Conclusion}

In this paper, we show how we applied the 4C/ID in developing deliberate practice to facilitate schema acquisition. More examples and assessment results will be presented in the poster.

\section{Acknowledgement}

This study is supported by the National Science Foundation under Grant Nos. 1927284 and 1927290. 


\section{References}

[1] J. Sweller, P. Ayres, and S. Kalyuga. Cognitive Load Theory. Explorations in the learning sciences, instructional systems and performance technologies: Vol. 1., 2011.

[2] K. A. Ericsson, "The Differential Influence of Experience, Practice, and Deliberate Practice on the Development of Superior Individual Performance of Experts," in The Cambridge Handbook of Expertise and Expert Performance, 2nd ed., K. A. Ericsson, R. R. Hoffman, A. Kozbelt, and A. M. Williams, Eds. Cambridge: Cambridge University Press, 2018, pp. 745-769.

[3] K. A. Ericsson, R. T. Krampe, and C. Tesch-Römer, "The role of deliberate practice in the acquisition of expert performance". Psychological review, 100(3), 363-406, 1993.

[4] Y. Tang and H. Bai, "Develop a better way to practice to enhance students' experience in learning dynamics," in ASEE Annual Conference \& Exposition, Seattle, Washington, 2015.

[5] Y. Tang, "Cognitive benefits of using the kinetic diagrams in teaching introductory dynamics," in ASEE Annual Conference \& Exposition, Tampa, Florida, 2019.

[6] C. A. Moulton, A. Dubrowski, H. Macrae, B. Graham, E. Grober, and R. Reznick, "Teaching surgical skills: what kind of practice makes perfect?: a randomized, controlled trial," Annals of surgery, 244(3), 400-409, 2006.

[7] K. A. Ericsson, "Acquisition and maintenance of medical expertise," Academic Medicine, 90(11), 1471-1486., 2015.

[8] S. Andersen; P. Mikkelsen, L. Konge, P. Caye-Thomassen, and M. S. Sørensen, "Cognitive load in distributed and massed practice in virtual reality mastoidectomy simulation," The Laryngoscope, 126(2), E74-E79, 2016.

[9] J. v. Merrienboer, P. A. Kirschner, Ten Steps to Complex Learning: A Systematic Approach to Four-Component Instructional Design. Rouledge, 2018. 